\title{
Halitosis and Associated Risk Factors in Children: A Cross-sectional Study
}

\author{
Noura A AlMadhi ${ }^{1}$, Ayman M Sulimany ${ }^{2}$, Hamad A Alzoman ${ }^{3}$, Omar A Bawazir ${ }^{4}$
}

\begin{abstract}
Aim: To investigate risk factors associated with halitosis in children using OralChroma ${ }^{\mathrm{TM}}$.

Materials and methods: Sixty-seven children between the ages of 3 and 8 who attended a pre-general anesthesia assessment at the Dental University Hospital at King Saud University, Riyadh, Saudi Arabia, were enrolled in this study after satisfying the inclusion criteria. Demographic data, medical history, and oral hygiene practices were obtained by a standardized questionnaire completed by the parents of each child. Clinical dental examination was conducted to record the following: caries index (dmft/DMFT), simplified debris index (DI-S), modified gingival index (MGI), and Winkel tongue coating index (WTCl). Clinical halitosis was assessed using the OralChroma ${ }^{\mathrm{TM}}$ device that measures the concentration of volatile sulfur compounds (VSCs) including hydrogen sulfide $\left(\mathrm{H}_{2} \mathrm{~S}\right)$, methyl mercaptan $\left(\mathrm{CH}_{3} \mathrm{SH}\right)$, and dimethyl sulfide $\left(\mathrm{CH}_{3} \mathrm{SCH}_{3}\right)$. Statistics were completed using Spearman's correlation coefficient and Mann-Whitney U-test to assess the association with the VSC scores with continuous and binary variables, respectively. Then, multivariate linear regression analysis was performed to detect the degree of association.

Results: High VSC measures were detected in 58 children (87\%). The dmft/DMFT was significantly associated with $\mathrm{H}_{2} \mathrm{~S}$ level $(\beta=26.84, p=0.034)$ and $\mathrm{CH}_{3} \mathrm{SH}$ level $(\beta=19.96, p=0.016)$ after controlling possible confounders. There was no significant association of DI-S, MGI, and WTCI with the VSC measures.

Conclusion: The result suggested that dmft/DMFT score (children's caries experience) is associated significantly with high levels of $\mathrm{H}_{2} \mathrm{~S}$ and $\mathrm{CH}_{3} \mathrm{SH}$ in the sample studied.

Clinical significance: Caries experience in children is a significant risk factor for halitosis and should be considered during the management of halitosis.

Keywords: Children, Dental caries, Halitosis, OralChroma, Volatile sulfur compounds.

The Journal of Contemporary Dental Practice (2021): 10.5005/jp-journals-10024-3017
\end{abstract}

\section{INTRODUCTION}

"Halitosis" is one of the terms used to describe oral bad breath that may relate to extra- or intra-oral factors, with the latter accounting for $90 \%$ of all cases. It has been reported as a common problem worldwide among adult and elderly populations. ${ }^{1}$ Halitosis also affects children with a prevalence ranged between 8 and $45 \% .^{2-6}$ An association was reported between halitosis and multiple risk factors in studies conducted on the pediatric population including caries, food impaction, poor oral hygiene, proximal restoration, gingival health, tongue coating, mouth breathing, and presence of orthodontic appliances. ${ }^{2,5-13}$ However, the results of these studies are inconsistent and contradictory due to the different clinical indices used and the different methods used to assess halitosis.

The most common methods used by researchers to assess halitosis in children are organoleptic assessment, ${ }^{3,6}$ sulfide monitors, ${ }^{11,14}$ or combining both methods. ${ }^{2,5,7,9-12}$ Organoleptic measurement was considered gold standard to measure halitosis because its simple method of conduction and cheapness. It requires no special equipment, and human judgment could be the most logical measurement method for perceived olfactory stimulus. ${ }^{15,16}$ However, this method raises several problems; mainly, the reliability of the examiner, the effect of the environment on the olfactory capacity of the examiner, the need for examiner training, and the potential risk for disease transmission via expelled air. ${ }^{11,15-17}$ A sulfide monitor is a portable device that measures total sulfur compounds in breaths and is mainly used to monitor the prognosis of oral halitosis rather than detect it because it cannot differentiate \begin{tabular}{l}
\hline \hline 1,2,4 Department of Pediatric Dentistry and Orthodontics, College of \\
Dentistry, King Saud University, Riyadh, Kingdom of Saudi Arabia \\
${ }^{3}$ Department of Periodontics and Community Dentistry, College of \\
Dentistry, King Saud University, Riyadh, Kingdom of Saudi Arabia \\
Corresponding Author: Noura A AIMadhi, Department of Pediatric \\
Dentistry and Orthodontics, College of Dentistry, King Saud University, \\
Riyadh, Kingdom of Saudi Arabia, Phone: +966 500097447, e-mail: \\
Nourahalmadhi@gmail.com \\
How to cite this article: AlMadhi NA, Sulimany AM, Alzoman HA, et al. \\
Halitosis and Associated Risk Factors in Children: A Cross-sectional \\
Study. J Contemp Dent Pract 2021;22(1):51-55. \\
Source of support: Nil \\
Conflict of interest: None
\end{tabular}

between different sulfur compounds. ${ }^{18}$ The other limitation of this method is the incorrect positive halitosis detection due to the presence of additional volatile vapors that do not cause halitosis like acetone, ethanol, or methanol. ${ }^{16}$ Currently, OralChroma ${ }^{\mathrm{TM}}$ is a commercially available type of gas chromatography, combining both the compact gas chromatography system and the semiconductor gas sensor that allow a quick measurement of volatile sulfur compounds (VSCs). ${ }^{19}$ It provides separate values for hydrogen sulfide $\left(\mathrm{H}_{2} \mathrm{~S}\right)$, methyl mercaptan $\left(\mathrm{CH}_{3} \mathrm{SH}\right)$, and dimethyl sulfide $\left(\mathrm{CH}_{3} \mathrm{SCH}_{3}\right)$ which represent $90 \%$ of VSCs and are considered the major compounds associated with halitosis. ${ }^{20}$ This device's reliability for assessing and diagnosing halitosis has been reported 
in many studies ${ }^{17}$ and is considered to be the gold standard when evaluating the VSC concentrations. ${ }^{21}$ One recent study showed a significant correlation between OralChroma ${ }^{\mathrm{TM}}$ with other evaluation methods of halitosis in children. ${ }^{13}$

In the literature, risk factors for oral origin associated with halitosis in children have been investigated with the use of either organoleptic and/or sulfide monitors. However, no study has investigated the oral risk factors associated with an increase in VSCs in children using OralChroma ${ }^{\mathrm{TM}}$. Therefore, this study was conducted to investigate the possible risk factors associated with halitosis in children using OralChroma ${ }^{\mathrm{TM}}$.

\section{Materials and Methods}

\section{Sample Selection and Ethical Approval}

This cross-sectional study was carried out during the period from January to September 2019. Sixty-seven children between the ages 3 and 8 years were recruited from patients who attended morning GA (general anesthesia) screening clinics at Dental University Hospital, King Saud University (KSU), Riyadh, Saudi Arabia. The inclusion criteria were healthy (ASA I and II), cooperative child during breath sample collection and initial clinical examination, with no history of upper respiratory tract infection, tonsillar infection, allergic rhinitis, Candida infection, gastrointestinal diseases, and no history of antibiotic usage during the last month before the appointment. The aim and the protocol of the study were explained to the parents one day before the screening appointment over the phone to obtain initial approval and to instruct parents regarding the breath sample test. All parents were told that their child should not eat or drink and refrain from brushing their teeth and using mouthwashes for a couple of hours before the breath sample collection. Patients were scheduled early in the morning and a written consent with all the details of the study was obtained from the parents of all participants. The study was approved by the ethical committee in KSU.

\section{Clinical Oral Examinations}

A self-administrated questionnaire was carried out first to obtain the demographic data and oral hygiene practices. Then, patients were examined on the dental chair by the primary investigator (nursing assistant) to collect the following data: dental caries score using dmft/DMFT (decayed, missing, and filled teeth) index by WHO (2013), ${ }^{22}$ oral hygiene level using simplified debris index (DI-S) by Greene and Vermillion (1964), ${ }^{23}$ gingival health condition using modified gingival index (MGI) by Lobene et al. (1986), ${ }^{24}$ tongue coating using Winkel tongue coating index (WTCI) by Winkel et al. (2003), ${ }^{25}$ and the number of abscessed teeth. Intraexaminer reliability was assessed before conducting the study on 10 children similar to the sample but not included in the study at 2-week intervals for the clinical variables (dmft/DMFT, DI-S, MGI, and WTCI) using correlation coefficient and all scores were above 0.7 .

\section{Measuring the Level of VSCs Using OralChroma ${ }^{\mathrm{TM}}$}

The breath sample procedures were explained to the parents and their child in the halitosis clinic using an instructional video. Children were instructed to close their mouths and breathe through the nose for 1 minute. Then, using a disposable plastic syringe, a breath sample of $1 \mathrm{~mL}$ was attained and injected immediately into the OralChroma ${ }^{\mathrm{TM}}$ device (Nissha FIS, Inc., Abimedical Corporation, Osaka, Japan). The concentration of gases $\mathrm{H}_{2} \mathrm{~S}, \mathrm{CH}_{3} \mathrm{SH}$, and $\mathrm{CH}_{3} \mathrm{SCH}_{3}$ was measured. Clinically, the present halitosis was considered when any of the VSCs is equal or higher than the following cognitive threshold according to the manufacturer's instructions: for $\mathrm{H}_{2} \mathrm{~S} \geq 112$, $\mathrm{CH}_{3} \mathrm{SH} \geq 26$, and $\mathrm{CH}_{3} \mathrm{SCH}_{3} \geq 8$, all in parts per billion.

\section{Sample Size and Statistical Analysis}

At the power of $90 \%$ and 0.8 effect size, the sample size was calculated with a 0.05 level of significance. ${ }^{10,26}$ A total of 67 children were included in the study. Data were analyzed with SPSS software (IBM Inc., Chicago, USA). Descriptive statistics were used, such as the mean and standard deviation or frequencies and percentages, to describe quantitative and categorical variables. Gingival health $(0=$ no inflammation, $1=$ inflamed $)$ and dental abscess ( $0=$ no abscess, $1=$ abscess present) were dichotomized for the purposes of analysis. The normalization of the data was tested using Kolmogorov-Smirnov and Shapiro test. The association of VSC levels with clinical variables was examined using Spearman's correlation coefficient for continuous variables and Mann-Whitney $U$-test for binary variables. Multivariable linear regression was used to detect the degree of association after controlling the possible confounding factors. The level of statistical significance was set at $p<0.05$.

\section{Results}

Sixty-seven Saudi children including 44 females and 23 males with a mean age of $5.3 \pm 1.7$ years participated in this study. Fifty-eight $(86.6 \%)$ children were reported to have clinical halitosis using OralChroma $^{\mathrm{Tm}}$. The mean \pm standard deviation and frequency of clinical variables are presented in Table 1. Mean \pm SD of dmft/DMFT and WTClare $11.3 \pm 3.3$ and $3.6 \pm 1.3$, respectively. Most of the samples had poor oral hygiene ( $n=47,70.1 \%)$, about half of the participants had gingival inflammation in varying degrees $(n=31,46.3 \%)$,

Table 1: Descriptive presentation of the clinical data for the children, $(n=67)$

\begin{tabular}{lrl}
\hline Clinical parameters & Mean \pm SD & Frequency (\%) \\
\hline Age & $5.3 \pm 1.7$ & \\
Caries index-total dmft/DMFT & $11.3 \pm 3.3$ & \\
Winkel tongue coating index & $3.6 \pm 1.3$ & \\
Debris index & $20(29.9)$ \\
- Fair & $47(70.1)$ \\
- Poor & \\
Modified gingival index & $36(53.7)$ \\
- Absence of inflammation & $23(43.1)$ \\
- Mild localized inflammation & $5(7.5)$ \\
$\quad$ inflammation & $1(1.5)$ \\
- Moderate inflammation & $2(3.0)$ \\
- Severe inflammation & \\
Gingival health & $36(53.7)$ \\
- No inflammation & $31(46.3)$ \\
Presence of dental abscesses & \\
- No & $32(47.8)$ \\
- Yes & $35(52.2)$ \\
Daily toothbrushing practice* & \\
• No $\quad$ Yes & $24(35.8)$ \\
\hline
\end{tabular}

*Obtained from questionnaire 
Table 2: Level of VSCs in correlation with clinical parameters, $(n=67)$

\begin{tabular}{|c|c|c|c|c|c|c|}
\hline Clinical parameters & $\mathrm{H}_{2} \mathrm{~S}$ & $p$ value & $\mathrm{CH}_{3} \mathrm{SH}$ & $p$ value & $\mathrm{CH}_{3} \mathrm{SCH}_{3}$ & $p$ value \\
\hline Caries index $(\mathrm{dmft}+\mathrm{DMFT})^{\$}$ & 0.275 & 0.025 & 0.248 & 0.043 & 0.190 & 0.124 \\
\hline Winkel tongue coating index $-\mathrm{WTCl}^{\$}$ & 0.131 & 0.292 & 0.104 & 0.401 & 0.167 & 0.178 \\
\hline $\begin{array}{l}\text { Debris index* } \\
\text { - } \quad \text { Fair } \\
\text { - } \quad \text { Poor }\end{array}$ & $\begin{array}{l}143(526.7) \\
225(260)\end{array}$ & 0.603 & $\begin{array}{l}26(129.5) \\
63(97)\end{array}$ & 0.238 & $\begin{array}{l}3(10.5) \\
10(39)\end{array}$ & 0.076 \\
\hline $\begin{array}{l}\text { Gingival health* } \\
\text { - No inflammation } \\
\text { - Inflammation }\end{array}$ & $\begin{array}{l}143(229.25) \\
235(657)\end{array}$ & 0.054 & $\begin{array}{l}36(81.75) \\
86(132)\end{array}$ & 0.031 & $\begin{array}{c}10(27.5) \\
9(39)\end{array}$ & 0.929 \\
\hline $\begin{array}{l}\text { Presence of dental abscesses* } \\
\begin{array}{l}\text { - No } \\
\text { - Yes }\end{array}\end{array}$ & $\begin{array}{l}137.5(303) \\
225(396)\end{array}$ & 0.572 & $\begin{array}{l}46.5(95.5) \\
62(123)\end{array}$ & 0.669 & $\begin{aligned} 8(22.3) \\
10(39)\end{aligned}$ & 0.402 \\
\hline $\begin{array}{l}\text { Daily toothbrushing practice* } \\
\text { - No } \\
\text { - Yes }\end{array}$ & $\begin{array}{l}130(198) \\
227(476)\end{array}$ & 0.059 & $\begin{array}{l}59(99.2) \\
51(126)\end{array}$ & 0.619 & $\begin{array}{r}11.5(41) \\
7(19)\end{array}$ & 0.181 \\
\hline
\end{tabular}

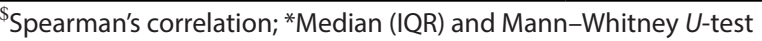

Table 3: Multiple linear regression summary of $\mathrm{H}_{2} \mathrm{~S}$ levels and clinical parameters

\begin{tabular}{lccc}
\hline & \multicolumn{3}{c}{$\mathrm{H}_{2} \mathrm{~S}$} \\
\cline { 2 - 3 } & \multicolumn{2}{c}{ Unstandardized coefficients } & \\
\cline { 2 - 3 } Variables & $B$ & Std. error & p value \\
\hline Caries index & 26.842 & 12.413 & 0.034 \\
(dmft + DMFT) & & & \\
$\begin{array}{l}\text { Gingival health } \\
\begin{array}{l}\text { Daily toothbrushing } \\
\text { practice }\end{array}\end{array}$ & 135.446 & 80.378 & 0.097 \\
\hline
\end{tabular}

Table 4: Multiple linear regression summary of $\mathrm{CH}_{3} \mathrm{SH}$ levels and clinical parameters

\begin{tabular}{lccc}
\hline & \multicolumn{2}{c}{$\mathrm{CH}_{3} \mathrm{SH}$} & \\
\cline { 2 - 3 } Variables & \multicolumn{2}{c}{ Unstandardized coefficients } & \\
\cline { 2 - 3 } & \multicolumn{1}{c}{ Caries index } & Std.error & p value \\
\hline $\begin{array}{l}\text { (dmft + DMFT) } \\
\text { Gingival health }\end{array}$ & 19.961 & 8.032 & 0.016 \\
\hline
\end{tabular}

and more than half of the samples had dental abscesses $(n=35$, $52.2 \%)$. Forty-three children (64.2\%) brushed their teeth daily according to their parents.

\section{Association of VSCs and Clinical Parameters}

The age and gender were assessed in association with VSC values, and no statistically significant association was found in the bivariate analysis. The association between VSC values and the clinical parameters presented in Table 2. Both $\mathrm{H}_{2} \mathrm{~S}$ and $\mathrm{CH}_{3} \mathrm{SH}$ mean concentrations were significantly associated with the dmft/ DMFT index and gingival health in the bivariate analysis. To confirm the degree of association of $\mathrm{H}_{2} \mathrm{~S}$ and $\mathrm{CH}_{3} \mathrm{SH}$ concentrations with potential risk factors, a multiple linear regression test was performed (Table 3 and 4. The dmft/DMFT index was associated significantly with a mean level of $\mathrm{H}_{2} \mathrm{~S}$ and $\mathrm{CH}_{3} \mathrm{SH}(p=0.03,0.02)$, respectively. In addition, daily toothbrushing practice was associated with the level of $\mathrm{H}_{2} \mathrm{~S}(p=0.04)$.

\section{Discussion}

It is widely believed nowadays that halitosis is caused by the increase of VSC concentrations in the oral cavity due to the action of anaerobic microorganisms by breaking down the components of the epithelial cells, salivary proteins, and food debris. ${ }^{16} \mathrm{The}_{2} \mathrm{~S}$ and $\mathrm{CH}_{3} \mathrm{SH}$ are believed to be the main VSCs that contribute to intraoral halitosis, while $\mathrm{CH}_{3} \mathrm{SCH}_{3}$ is mainly linked with extraoral halitosis. ${ }^{27}$ In this study, the $\mathrm{CH}_{3} \mathrm{SH}$ and $\mathrm{H}_{2} \mathrm{~S}$ levels were higher than that of $\mathrm{CH}_{3} \mathrm{SCH}_{3}$, which is consistent with earlier studies that support the high concentration of both gases in halitosis of oral origin. ${ }^{13,20}$

The present study showed a high prevalence of halitosis in comparison to other studies. ${ }^{3,5,6,9,10,13}$ This finding is not surprising as the halitosis was measured objectively using the OralChroma ${ }^{\text {TM }}$ device that can detect a low concentration of VSC molecules ${ }^{28}$ unlike other studies that evaluated halitosis subjectively using the organoleptic technique, ${ }^{3,6}$ which also carries the risk of transmission of infectious disease or used sulfide monitors that cannot detect all halitosis-causing VSCs. ${ }^{11,14}$ Also, the sample selected in this study was from children with a high caries index who are on the waiting list for GA due to their uncooperative behavior to provide treatment on the dental chair or due to extensive dental needs. Additionally, the time of breath sampling collection may have influenced the results as previous studies showed an increase in halitosis scores when the breath was evaluated in the morning. ${ }^{15,29}$

In the current study, the correlation between the children's caries experience (dmft/DMFT) with $\mathrm{H}_{2} \mathrm{~S}$ and $\mathrm{CH}_{3} \mathrm{SH}$ levels was statistically significant, but not with $\mathrm{CH}_{3} \mathrm{SCH}_{3}$. This finding is in agreement with the literature that shows that the main contributors to intraoral halitosis are believed to be $\mathrm{H}_{2} \mathrm{~S}$ and $\mathrm{CH}_{3} \mathrm{SH}$, while $\mathrm{CH}_{3} \mathrm{SCH}_{3}$ is mainly associated with extraoral halitosis. ${ }^{27}$

This result is consistent with Gholami et al. who reported relation of dental caries with halitosis, while other studies did not find the same association. ${ }^{3,5-8,10,15}$ The difference between this finding and the previous studies is related to differences in sample size, age, evaluation methods, and DMFT scores. For example, Amir et al. and Nalçaci et al. found no association using a smaller sample with a wider age range (5-14 and 7-15 years, respectively) and lower DMFT scores compared to this study. 7,10 Also, no association was reported by Kanehira et al. who evaluated 
children less than 5 years with lower decay scores. ${ }^{5}$ Similarly, Ueno et al. and Patil et al. found no association in their samples, which consisted of school-age children with a higher proportion of caries-free children. ${ }^{3,6}$

The present study did not find an association between VSCs and tongue coating, which agrees with Lin et al.'s study. ${ }^{9}$ However, a relationship between halitosis and tongue coating was found in many studies conducted on children. ${ }^{3,6,7,10}$ It is worth mentioning that two of these studies reported the impact of tongue cleaning on halitosis and showed contradictory results. ${ }^{9,29}$ Lin et al. evaluated two tongue cleaning methods on VSCs using a Halimeter and noticed an increase in VSC levels postcleaning in both groups and attributed that to a gag reflex induced by cleaning the tongue, which resulted in a pharyngeal breath that increased VSC scores. ${ }^{9}$ On the contrary, Kara et al. noted a positive effect of tongue hygiene on halitosis which was part of an intervention including scaling and oral hygiene; however, tongue coating score pre- and postintervention were not mentioned. ${ }^{29}$

The relationship between halitosis and oral hygiene (debris index) and gingival health in this study was not significant, as more than half of the children had no inflammation which is in agreement with the previous studies ${ }^{5,6,9}$ in contrast to others. ${ }^{2,3,7,10}$ Studies supporting an association of plaque, gingival, and periodontal health were conducted on patients who originally complained of halitosis ${ }^{7,10}$ or who were caries free and evaluated to figure out the risk factors ${ }^{2}$ or children from an older age-group. ${ }^{3}$

The association of oral hygiene practice showed a significant association with $\mathrm{H}_{2} \mathrm{~S}$ levels. This result was surprising, as it showed children who brush their teeth daily have higher levels; the plausible explanation for this result may be the lack of manual dexterity in this age-group or social desirability bias. Similar to other factors, studies showed contradictory results that either reported an association between the frequency of toothbrushing and halitosis ${ }^{3,7}$ or no association. . $^{5,9,9,10}$

Half of the children in the present study have dental abscesses; however, abscesses were not associated with VSCs. The only explanation for this is that certain odors do not contribute to VSC levels or cannot be measured by gas chromatography. ${ }^{30}$

In summary, the results of studies on the associated factors with halitosis in children are not consistent and inconclusive. It should be taken into account that the objectives, sampling, evaluation methods of halitosis, indices used for clinical parameters, and statistical methods differ between studies. However, it can still be explained that studies conducted on caries-free, low caries prevalence children or children complaining of halitosis show that usually oral hygiene, gingival and periodontal status, and tongue coating play significant roles in halitosis. On the contrary, children with a high caries index associate significantly with higher concentrations of VSCs. On the contrary, children with a high caries index associate significantly with higher concentrations of VSCs.

The limitations in this study are as follows: the sample was not randomly selected and not representative of the population and did not include children with a low caries index. The positive points of this study are the use of the most objective method in measuring halitosis (OralChroma ${ }^{\text {TM}}$ ) to assess the effect of clinical parameters on VSC measurements and to limit the study to a small and young age-group (from 3 to 8 years). Further research should be conducted on different age-groups to assess the risk factors, compare cariesfree/low caries and high caries children, and investigate the impact of full dental rehabilitation on the VSC measurements.

\section{Conclusion}

In this study, the dmft/DMFT index (children's caries experience) was associated significantly with intraoral halitosis caused by high $\mathrm{H}_{2} \mathrm{~S}$ and $\mathrm{CH}_{3} \mathrm{SH}$ levels. Other clinical parameters, such as DI-S, MGI, WTCl, and dental abscesses were not associated with higher VSCs measurements in children.

\section{References}

1. Cortelli JR, Dourado M, Barbosa S, et al. Halitosis: a review of associated factors and therapeutic approach. Braz Oral Res 2008;442222(1):44-5444. DOI: 10.1590/S1806-83242008000500007.

2. Villa $A$, Zollanvari A, Alterovitz $G$, et al. Prevalence of halitosis in children considering oral hygiene, gender and age. Int J Dent Hyg 2014;12(3):208-212. DOI: 10.1111/idh.12077.

3. Patil PS, Pujar P, Poornima S, et al. Prevalence of oral malodour and its relationship with oral parameters in Indian children aged 7-15 years. Eur Arch Paediatr Dent 2014;15(4):251-258. DOI: 10.1007/s40368-014-0109-0.

4. Yoshida M, Takamori K, Ono Y, et al. Study on halitosis children: questionnaire and halitosis test. Pediatr Dent J 2001;39:694-703. DOI: 10.11411/jspd1963.39.3_694.

5. Kanehira T, Takehara J, Takahashi D, etal. Prevalence of oral malodor and the relationship with habitual mouth breathing in children.J Clin Pediatr Dent 2004;28(4):285-288. DOI: 10.17796/jcpd.28.4.xp213r6534322m58.

6. Ueno $M$, Ohnuki $M$, Zaitsu $T$, et al. Prevalence and risk factors of halitosis in Japanese school children. Pediatr Int 2018;60(6):588-592. DOI: 10.1111/ped.13561.

7. Amir E, Shimonov R, Rosenberg M. Halitosis in children. J Pediatr 1999;134(3):338-343. DOI: 10.1016/s0022-3476(99)70460-9.

8. Gholami F, Minah GE, Turng BF. Oral malodor in children and volatile sulfur compound-producing bacteria in saliva: preliminary microbiological investigation. Pediatr Dent 1999;21(6):320-324. Available from: https:// www.aapd.org/globalassets/media/publications/archives/paryavigholam-21-06.pdf.

9. Lin MIH, Flaitz CM, Moretti AJ, et al. Evaluation of halitosis in children and mothers. Pediatr Dent 2003;25(6):553-558. Available from: https://www.cnzscx.org/globalassets/media/publications/archives/ lin-25-06.pdf.

10. Nalçaci R, Sönmez IS. Evaluation of oral malodor in children. Oral Surg Oral Med Oral Pathol Oral Radiol Endodontol 2008;106(3):384-388. DOI: 10.1016/j.tripleo.2008.03.001.

11. Motta LJ, Bachiega JC, Guedes CC, et al. Association between halitosis and mouth breathing in children. Clinics 2011;66(6):939-942. DOI: 10.1590/S1807-59322011000600003.

12. Keceli T, Gulmez D, Dolgun A, et al. The relationship between tongue brushing and halitosis in children: a randomized controlled trial. Oral Dis 2015;21(1):66-73. DOI: 10.1111/odi.12210.

13. Costacurta $M$, Petrini $M$, Biferi $V$, et al. The correlation between different techniques for the evaluation of oral malodour in children with and without orthodontic treatment. Eur J Paediatr Dent 2019;20(3):233-236. DOI: 10.23804/ejpd.2019.20.03.12.

14. Babacan H, Sokucu O, Marakoglu I, et al. Effect of fixed appliances on oral malodor. Am J Orthod Dentofac Orthop 2011;139(3):351-355. DOI: 10.1016/j.ajodo.2009.03.055.

15. Miyazaki H, Sakao S, Katoh $\mathrm{Y}$, et al. Correlation between volatile sulphur compounds and certain oral health measurements in the general population. J Periodontol 1995;66(8):679-684. DOI: 10.1902/ jop.1995.66.8.679.

16. Lee PPC, Mak WY, Newsome P. The aetiology and treatment of oral halitosis : an update. Hong Kong Med J 2004;10(6):414-418. Available from: https://www.hkmj.org/system/files/hkm0412p414.pdf.

17. Tangerman A, Winkel EG. The portable gas chromatograph OralChroma ${ }^{\mathrm{TM}}$ : a method of choice to detect oral and extra-oral halitosis. J Breath Res 2008;2(1). DOI: 10.1088/1752-7155/2/1/017010.

18. Hughes FJ, McNab R. Oral malodour-a review. Arch Oral Biol 2008;53(1):1-7. DOI: 10.1016/S0003-9969(08)70002-5. 
19. Hanada $\mathrm{M}$, Koda $\mathrm{H}$, Onaga $\mathrm{K}$, et al. Portable oral malodor analyzer using highly sensitive $\ln _{2} \mathrm{O}_{3}$ gas sensor combined with a simple gas chromatography system. Anal Chim Acta 2003;475(1-2):27-35. DOI: 10.1016/S0003-2670(02)01038-3.

20. Lee $\mathrm{CH}$, Kho HS, Chung SC, et al. The relationship between volatile sulfur compounds and major halitosis-inducing factors. J Periodontol 2003;74(1):1-6. DOI: 10.1902/jop.2003.74.1.32.

21. Yaegaki K, Coil JM. Examination, classification, and treatment of halitosis; clinical perspectives. J Can Dent Assoc 2000;66(5):257261. DOI: 10.1002/j.1875-595X.2002.tb00921.x.

22. World Health Organization. Oral health surveys: basic methods - 5th edition. http://www.who.int/oral_health/ publications/9789241548649/en/.

23. Greene JC, Vermillion Jr. the Simplified Oral Hygiene Index. J Am Dent Assoc 1964;68(1):7-13. DOI:10.14219/jada.archive.1964.0034.

24. Rebelo MAB, Queiroz AC de. Gingival Indices: State of Art, Gingival Diseases - Their Aetiology, Prevention and Treatment. Gingival Dis Their Aetiol Prev Treat. Published online 2011:246. doi:10.5772/26236.

25. Winkel EG, Roldán S, Van Winkelhoff AJ, et al. Clinical effects of a new mouthrinse containing chlorhexidine, cetylpyridinium chloride and zinc-lactate on oral halitosis: a dual-center, double-blind placebo- controlled study. J Clin Periodontol 2003;30(4):300-306. DOI 10.1034/j.1600-051X.2003.00342.x.

26. Schemel-suárez M, Chimenos-küstner E, Estrugo-devesa $A$, et al. Halitosis assessment and changes in volatile sulfur compounds after chewing gum: a study performed on dentistry students. J Evid Based Dent Pract 2017;17(4):381-388. DOI: 10.1016/j. jebdp.2017.06.001.

27. Tangerman A, Winkel E. Intra- and extra-oral halitosis: finding of a new form of extra-oral blood-borne halitosis caused by dimethyl sulphide. J Clin Periodontol 2007;34(9):748-755. DOI: 10.1111/j.1600051X.2007.01116.x.

28. Aylıkı BU. Halitosis: from diagnosis to management. J Nat Sci Biol Med 2013;4(1):14-23. DOI: 10.4103/0976-9668.107255.

29. Kara C, Tezel A, Orbak R. Effect of oral hygiene instruction and scaling on oral malodour in a population of Turkish children with gingival inflammation. Int J Paediatr Dent 2006;16(6):399-404. DOI: 10.1111/j.1365-263X.2006.00769.x.

30. van den Broek AMWT, Feenstra $L$, de Baat $C$. A review of the current literature on aetiology and measurement methods of halitosis. J Dent 2007;35(8):627-635. DOI: 10.1016/j.jdent.2007.04.009. 